\title{
Corela
}

Cognition, représentation, langage

HS-34 | 2021

Les noms sous-spécifiés en français: du lexique au discours

\section{Les noms sous-spécifiés en français : du lexique au discours. Présentation}

Dejan Stosic et Josette Rebeyrolle

\section{(2) OpenEdition}

Journals

Édition électronique

URL : https://journals.openedition.org/corela/13467

DOI : $10.4000 /$ corela. 13467

ISSN : 1638-573X

Éditeur

Cercle linguistique du Centre et de l'Ouest - CerLICO

Référence électronique

Dejan Stosic et Josette Rebeyrolle, « Les noms sous-spécifiés en français : du lexique au discours. Présentation », Corela [En ligne], HS-34 | 2021, mis en ligne le 08 décembre 2021, consulté le 13 décembre 2021. URL : http://journals.openedition.org/corela/13467 ; DOI : https://doi.org/10.4000/ corela.13467

Ce document a été généré automatiquement le 13 décembre 2021.

\section{(†)(2)

Corela - cognition, représentation, langage est mis à disposition selon les termes de la licence Creative Commons Attribution - Pas d'Utilisation Commerciale - Partage dans les Mêmes Conditions 4.0 International. 


\title{
Les noms sous-spécifiés en français : du lexique au discours. Présentation
}

\author{
Dejan Stosic et Josette Rebeyrolle
}

$1 \mathrm{Si}$, dans son ensemble, le lexique nominal du français a fait l'objet de nombreuses recherches en linguistique depuis plusieurs décennies (cf. Flaux \& Van de Velde 2000, Huyghe (éd.) 2015), certaines classes de noms restent encore relativement mal circonscrites. Tel est le cas des noms dits « sous-spécifiés » (NSS) (Winter 1992, Legallois 2006, 2008) comme idée, fait, proposition, principe, problème, dont les correspondants en anglais ont attiré l'attention des linguistes dès la fin des années 1960 (cf. Vendler 1968, Halliday \& Hasan 1976, Winter 1977, 1992). Les NSS se caractérisent par un contenu sémantique très pauvre, d'où les appellations «coquilles conceptuelles» (conceptual shells ou shell nouns, cf. Schmid 2000), « noms contenants » (container nouns, cf. Vendler 1968), «noms étiquettes» (labelling nouns, cf. Francis 1994, Partington 1998), «noms indices» (signaling nouns, cf. Flowerdew 2002, Flowerdew \& Forest 2015) ou encore «noms porteurs» (carrier nouns, cf. Ivanič 1991). Du fait de leur sous-spécification sémantique, ces noms ont une vaste application référentielle, et surtout la capacité à se charger du contenu sémantique en discours en «encapsulant» un contenu propositionnel plus ou moins large, soit par anaphore (ex. La direction a proposé au personnel de dormir sur place. L'idée ne ravit pas les syndicats.), soit par cataphore (ex. Le problème, c'est que nous sommes très différents.). En découle directement un rôle structurant important au niveau discursif, souligné par de nombreux auteurs (cf. Halliday \& Hasan 1976, Francis 1994, Schmid 2000, Legallois 2006, Rebeyrolle \& PéryWoodley 2014, Rebeyrolle 2015, Flowerdew \& Forest 2015). Dotés d'un contenu lexicosémantique et d'un fonctionnement discursif complexes, les NSS se retrouvent à la croisée de plusieurs autres classes nominales : les noms généraux (cf. Halliday \& Hasan 1976, Adler \& Moline (éds) 2018), les noms sommitaux (cf. Kleiber \& Lammert (éds) 2012), les noms d'idéalités (cf. Flaux \& Stosic 2015), et bien d'autres.

2 Le présent numéro fait suite à un colloque sur ce thème, organisé à l'Université Toulouse Jean Jaurès en novembre 2018. Cette rencontre a permis de réunir une vingtaine de linguistes travaillant sur cette sous-classe nominale relativement peu 
explorée et venant d'horizons méthodologiques et théoriques différents. Les études rassemblées dans ce numéro proposent d'une part un état de l'art sur ce thème et exposent d'autre part des résultats originaux et de nouvelles pistes de recherche en vue d'une meilleure circonscription de cette sous-classe nominale. Plus précisément, le numéro s'articule autour de trois axes principaux.

Un premier ensemble de travaux se proposent de préciser la sous-classe même des NSS, soit en l'opposant à d'autres sous-classes nominales soit en l'explorant de l'intérieur.

4 Le volume s'ouvre par la contribution de Richard Huyghe qui explore différents types de relations entre les NSS et les noms généraux (NG), dans le but de préciser la notion même de sous-spécification sémantique. L'auteur étudie les deux principales propriétés de celle-ci, régulièrement convoquées pour caractériser les deux classes de noms: la pauvreté du contenu sémantique et la position dominante dans la hiérarchie lexicale, d'une part, la nécessité de spécifier leur contenu en intégrant des éléments contextuels, d'autre part. L'article se clôt par une étude de cas du nom fait en français, considéré par R. Huyghe comme ayant un double statut de NG et de NSS.

5 Paul Capeau et Catherine Schnedecker poursuivent la confrontation des NG et des NSS au travers du prisme des noms d'humains dits généraux (NHG) comme homme, individu, personne, qui, bien que figurant très haut dans les hiérarchies lexicales, sont généralement et curieusement écartés du débat sur la sous-spécification sémantique. Manipulant un large panel de critères (usage, fréquence d'emploi, degré d'abstraction, potentiel de construction et de grammaticalisation, etc.), les auteurs mettent en lumière à la fois l'originalité des NHG au sein des NG et leur légitimité dans les discussions sur la notion de sous-spécification.

6 Les contours de la classe des NSS sont ensuite explorées par Marco Fasciolo par le biais des noms dits sommitaux comme chose, objet, lieu, personne, action, événement et rôles sémantiques généraux comme agent, patient, expérienceur. L'auteur montre toute la complexité des relations entre ces trois catégories et/ou objets d'étude qui, tout en manifestant une sorte de sous-spécification, apportent chacun(e) un éclairage particulier lorsqu'il s'agit d'étudier les fondements du lexique. C'est ce qui amène $M$. Fasciolo à proposer et à défendre une conception plus large des NSS, incluant les noms sommitaux.

7 Dans leur contribution, Dejan Stosic et Nelly Flaux s'intéressent à l'intersection entre les NSS et les noms dits d'idéalités, qui désignent des objets interprétables. Les auteurs partent en effet du constat qu'un bon nombre de noms comme idée, proposition, décision, principe, etc. sont considérés comme appartenant aux deux classes nominales. Après avoir mis au jour plusieurs propriétés sémantico-syntaxiques communes à celles-ci, D. Stosic et $\mathrm{N}$. Flaux tentent de déterminer les facteurs qui motivent le double classement de tels noms. La capacité à introduire un complément propositionnel apparaissant comme décisive, les auteurs interrogent d'une part la gamme des constructions syntaxiques éligibles, d'autre part leurs relations avec le nom-tête les introduisant.

8 Les deux dernières études du premier axe interrogent la classe des NSS de l'intérieur. En effet, considérant que la modalité est le trait sémantique principal des NSS, Dominique Legallois et Anaïs Vajnovszki proposent une classification modale de ces noms. Cette classification, inspirée des travaux de Chevalier et Léard (1993 et 1994) et de Gosselin $(2010,2015,2018)$, s'appuie sur un élargissement du champ de la modalité. 
L'objectif de cette nouvelle classification est de mieux rendre compte du fonctionnement sémantique, discursif et textuel des NSS.

Enfin, le travail présenté par Éric Laporte, Christiane Marque-Pucheu et Takuya Nakamura est un travail de révision de tables du lexique-grammaire de noms prédicatifs qui se donne pour objectif de mettre au jour ceux de ces noms qui sont susceptibles d'être employés comme NSS dans des constructions syntaxiques particulières. L'approche syntactico-sémantique défendue par les auteurs permet d'aborder une question nouvelle concernant les NSS : la nature prédicative du nom sous-spécifié, et le statut argumental ou non du complément phrastique dans une construction copulative spécificationnelle.

Le deuxième axe est constitué par six contributions qui sont toutes des études de cas. Chacune d'entre elles propose ainsi une analyse approfondie du fonctionnement d'un (ou de quelques) nom(s) sous-spécifié(s) dans différentes constructions dites spécificationnelles.

11 La première contribution de cette série, celle de Danièle Van de Velde, propose une description des propriétés sémantiques et pragmatiques du nom fait à partir d'une analyse des structures syntaxiques dans lesquelles il entre au titre de nom sousspécifié. Appuyant l'idée d'Adler (2017) que les NSS ne participent d'aucune " hiérarchie-être », D. Van de Velde propose de subdiviser la catégorie des NSS en trois sous-classes sur la base de critères syntaxiques. L'autrice montre ainsi que toutes les structures dites spécificationnelles ne sont pas identiques, et que le nom fait occupe, dans sa sous-classe d'appartenance, une place à part, tout comme au sein des NSS en général.

12 L'étude de Marie Lammert porte sur un autre nom présentant un haut degré de généralité et dont le statut linguistique exige d'être discuté, le nom catégorie. L'autrice montre que catégorie peut être considéré comme un NG ou bien comme un NSS selon que l'on s'appuie plutôt sur ses propriétés sémantiques (position lexicale, nonautonomie référentielle, sous-détermination intrinsèque et abstraction) ou syntaxiques. La mise en relation des propriétés sémantiques de catégorie avec ses caractéristiques syntaxiques ne permet finalement pas de conclure à l'inclusion de catégorie dans la classe des NSS.

Dans sa contribution consacrée au nom élément, Francine Gerhard-Krait défend l'hypothèse que la sous-spécification sémantique est à considérer comme faisant partie intégrante du sens lexical de certains noms, et non comme un simple effet d'usage en discours. Il est ainsi montré que le nom élément est par son essence même sous-spécifié, à cause de son caractère relationnel tout d'abord, mais aussi pour beaucoup d'autres raisons lexico-sémantiques et fonctionnelles mises en lumière par F. Gerhard-Krait.

14 L'étude de Céline Benninger porte sur un nom particulier, le nom chose, et se donne pour objectif d'examiner l'implication de ce nom dans la cohésion textuelle. Après avoir rappelé ses propriétés sémantiques ainsi que les caractéristiques de ses emplois, l'autrice examine le nom chose dans des configurations particulières où, en tant que noyau de syntagme démonstratif, il occupe la place de pivot autour duquel s'installent deux séquences interdépendantes. Elle présente les paramètres d'ordre sémanticopragmatique et syntaxique qui doivent être respectés pour pallier la pauvreté sémantique de ce nom.

Dans la continuité de la réflexion sur le fonctionnement du nom chose, Estelle Moline aborde dans sa contribution la polysémie du nom truc, qui présenterait au moins cinq 
acceptions différentes. Celles-ci sont établies à partir d'un vaste corpus d'exemples attestés et à travers une étude lexico-syntaxique approfondie de la combinatoire de truc avec quatorze structures syntaxiques invoquées dans la littérature sur les NSS. Cela permet à $\mathrm{E}$. Moline de mettre en évidence le caractère sous-spécifié plus ou moins prononcé de ce nom en fonction du type d'emploi où il apparaît.

Le dernier article du deuxième volet traite du nom émotion. Partant du constat d'un sémantisme peu spécifié qui a conduit de nombreux linguistes à qualifier ce nom de général, Emilia Hilgert montre qu'au-delà d'un emploi hypéronymique, ce nom connaît en français contemporain un usage qui en fait un nom de base, du même niveau lexical que peur, colère ou joie. La démonstration s'appuie sur un ensemble de propriétés linguistiques variées : morphologiques, syntaxiques et discursives.

17 Le troisième volet thématique comprend des contributions qui explorent les noms sous-spécifiés dans des corpus authentiques, à l'oral et à l'écrit.

Dans son article, Frédéric Sabio s'interroge sur l'attestation de tournures du type [N est c'est $\mathrm{X}]$, tels que le problème est c'est qu'il est parti, en français moderne. Après avoir comparé ces tournures non canoniques avec les constructions spécificationnelles du français standard, telles que le problème est qu'il est parti ou le problème c'est qu'il est parti, l'auteur s'intéresse aux types de NSS qu'elles semblent privilégier.

Le point de vue sous lequel Georgeta Cislaru aborde les NSS est celui de leur actualisation en discours et plus précisément dans des textes particuliers que sont les rapports éducatifs de la protection de l'enfance d'un côté et les dossiers académiques des étudiants de Master de l'autre. L'objectif de son étude est avant tout d'observer le rôle des NSS dans la structuration des dynamiques discursives propres à ces textes grâce à l'enregistrement en temps réel du processus ayant conduit à leur rédaction.

Les études réunies dans ce numéro fournissent un tableau inédit de la sous-classe nominale des NSS en français en articulant les enjeux théoriques de la délimitation d'une telle classe et les études fines et détaillées du comportement de certains noms particuliers. En même temps qu'elles mettent au jour des éléments nouveaux permettant une meilleure compréhension du fonctionnement de cette sous-classe nominale, nous espérons également que ces études ouvrent des pistes de recherche originales pour l'exploration future des NSS.

\section{BIBLIOGRAPHIE}

Adler, S. (2017). « Les noms généraux -« Shell Nouns »- participent-ils à une lecture taxinomique de type hiérarchie-être ?». Syntaxe et Sémantique 18, 45-66.

Adler, S. \& Moline, E. (éds) (2018). Les noms généraux. Langue française 198.

Chevalier, G. \& Léard, J.-M. (1994). « Le nom subordonnant : les séquences [SN que P] et la modalisation ». Travaux de Linguistique 27, 43-64. 
Chevalier, G. \& Léard, J.-M. (1996). « La subordination nominale : classes, sous-classes et types sémantiques ». Linguistische Arbeiten 351, 53-65.

Flaux, N. \& Stosic, D. (2015). « Pour une classe des noms d'idéalités », Langue française 185, 43-57.

Flaux, N. \& Van de Velde, D. (2000). Les noms en français, esquisse de classement. Paris, Gap : Ophrys.

Flowerdew, J. (2002). « A pedagogic grammar of signalling nouns in discourse ». Revista Canaria de Estudios Ingleses 44, 141-155.

Flowerdew, J. \& Forest, R. (2015). Signalling Nouns in English : A Corpus-based Discourse Approach. cambridge : cambridge University Press

Francis, G. (1994). « Labelling Discourse: An Aspect of Nominal-Group Lexical Cohesion ». In M. Coulthard (ed.), Advances in Written Text Analysis. London - New York : Routledge, 83-101.

Gosselin, L. (2010). Les modalités en français : la validation des représentations. Leyde (Pays-Bas) : Éditions Brill.

Gosselin, L. (2015). « De l'opposition modus/dictum à la distinction entre modalités extrinsèques et modalités intrinsèques ». Bulletin de la Société de Linguistique de Paris CX-1, 1-50.

Gosselin, L. (2018). « Quand nommer, c'est juger. Les jugements de valeur internes aux noms d'humains ». In C. Schnedecker (éd.), Nouvelles approches en sémantique lexicale : les noms d'humains, théories, méthodologies. Berlin : Mouton de Gruyter, 44-101.

Halliday, M.A.K. \& Hasan, R. (1976). Cohesion in English. London: Longman.

Huyghe, R. (éd.) (2015). « Les types nominaux ». Langue française 185.

Ivanič, R. (1991). « Nouns in search of a context ». International Review of Applied Linguistics $\operatorname{XXIX(2),~93-114.~}$

Kleiber, G. \& Lammert, M. (éds) (2012). « Questions de sémantique nominale ». Scolia 26.

Legallois, D. (2006). « Quand le texte signale sa structure : la fonction textuelle des noms sousspécifiés ». Corela: numéro thématique Organisation des textes et cohérence des discours. <http:// corela.revues.org/1465>

Legallois, D. (2008). « Sur quelques caractéristiques des noms sous-spécifiés ». Scolia 23, 109-127.

Partington, A. (1998). Patterns and Meanings. Using Corpora for English Language Research and Teaching. Amsterdam : John Benjamins Publishing.

Rebeyrolle, J. (2015). « Sémantique des noms dans les structures énumératives ». In M. VinaverKovic \& V. Stanojevic (éds), Les Études françaises aujourd'hui 2014. Belgrade : Faculté de Philologie de l'Université de Belgrade, 97-107.

Rebeyrolle, J. \& Péry-Woodley, M.-P. (2014). « Énumération et structuration discursive ». Actes du 4 e Congrès Mondial de Linguistique Française (CMLF 2014), Berlin, 19-23 juillet 2014, 3183-3196.

Schmid, H.J. (2000). English Abstract Nouns as Conceptual Shells: From Corpus to Cognition. Berlin New York, Walter de Gruyter.

Vendler, Z. (1968). Adjectives and nominalizations. The Hague : Mouton.

Winter, E. O. (1977). «A clause relational approach to English texts : a study of some predictive lexical items in written discourse ». Instructional Science 6, 1-92. 
Winter, E. O. (1992). «The notion of unspecific versus specific as one way of analyzing the information of a fund-raising letter ». In W. Mann \& S. A. Thompson (eds), Discourse description : diverse linguistic analyses of a fund-raising text. Amsterdam : John Benjamins Publishing, 131-170.

\section{AUTEURS}

DEJAN STOSIC

CLLE (UMR 5236 CNRS \& Université Toulouse Jean Jaurès)

JOSETTE REBEYROLLE

CLLE (UMR 5236 CNRS \& Université Toulouse Jean Jaurès) 